OPEN ACCESS

Edited by:

Hans-Jochem Kolb, Kolb Consulting UG, Germany

Reviewed by: Eric Spierings, Utrecht University, Netherlands Jakob Nilsson,

University Hospital Zürich, Switzerland

*Correspondence: Grigory A. Efimov efimov.g@blood.ru

Specialty section:

This article was submitted to Alloimmunity and Transplantation,

a section of the journal

Frontiers in Immunology

Received: 20 July 2018 Accepted: 14 May 2019

Published: 04 June 2019

Citation:

Romaniuk DS, Postovskaya AM Khmelevskaya AA, Malko DB and Efimov GA (2019) Rapid Multiplex

Genotyping of 20 HLA-A*02:01 Restricted Minor Histocompatibility Antigens. Front. Immunol. 10:1226.

doi: 10.3389/fimmu.2019.01226

\section{Rapid Multiplex Genotyping of 20 HLA-A*02:01 Restricted Minor Histocompatibility Antigens}

\author{
Dmitrii S. Romaniuk, Anna M. Postovskaya, Alexandra A. Khmelevskaya, Dmitry B. Malko \\ and Grigory A. Efimov*
}

Laboratory for Transplantation Immunology, National Research Center for Hematology, Moscow, Russia

A subset of MHC-associated self-peptides presented by the recipient's cells and immunologically foreign to the donor can induce an allogeneic immune response after hematopoietic stem cell transplantation (HSCT). These immunogenic peptides originate from the genomic polymorphisms and are known as minor histocompatibility antigens (MiHA). MiHA mismatches trigger the post-transplant immune response, which could manifest in both the deleterious "graft-vs.-host" disease and the beneficial "graft-vs.-leukemia" effect. Importantly, some MiHAs are considered to be promising targets for posttransplant T-cell immunotherapy of hematopoietic malignancies. This creates a demand for a robust and fast approach to genotyping MiHA-encoding polymorphisms. We report a multiplex real-time PCR method for the genotyping of 20 polymorphisms that are encoding HLA-A*02:01-restricted MiHAs. This method uses allele-specific primers and gene-specific hydrolysis probes. In $1 \mathrm{~h}$ it allows for the detection of MiHA mismatches in a donor-recipient pair without the need for electrophoresis, sequencing, or other time-consuming techniques. We validated the method with Sanger and NGS sequencing and demonstrated good performance over a wide range of DNA concentrations. We propose our protocol as a fast and accurate method of identifying mismatched MiHAs. The information on the MiHA mismatches is useful for studying the allogeneic immune response following HSCT and for selecting the targets for post-transplant T-cell therapy.

Keywords: multiplex qPCR, minor histocompatibility antigens, MiHA, SNP genotyping, allele-specific primer, AS-PCR, HSCT, AS-qPCR

\section{INTRODUCTION}

Allogeneic hematopoietic stem cell transplantation (allo-HSCT) is commonly used as a treatment for acute leukemias, lymphomas and other malignant hematopoietic diseases. The therapeutic efficiency of HSCT is determined by immune recognition and subsequent elimination of the remaining malignant clone by the infused lymphocytes of the donor origin, or so-called "graft-vs.-leukemia" (GvL) effect (1). Unfortunately, donor lymphocytes can also recognize and target some healthy non-hematopoietic tissue antigens, triggering potentially lethal "graft-vs.-host" disease (GvHD) (2).

The targets of the alloreactive immune response in the HLA-matched allo-HSCT are the minor histocompatibility antigens (MiHA). MiHAs are endogenous polymorphic peptides, presented by $\mathrm{MHC}$ molecules on the cell surface $(3,4)$. Donor $\mathrm{T}$ cells were not selected to tolerate 
the recipient's MiHA alleles, thus some transplanted Tcell clones may recognize them as foreign antigens $(5,6)$. Most MiHAs originate from non-synonymous single-nucleotide polymorphisms (nsSNP), however, they can be derived from other polymorphism types. Frameshifts and nonsense mutations result in the expression of truncated proteins, so only one of the allelic variants encodes a peptide, presented by MHC (7). Even a single amino acid substitution, caused by missense SNP, can affect peptide cleavage by the proteasome (8) or the antigen processing, resulting in the unilateral presentation of one of the allelic variants (9). The SNP variants encoding the presented and non-presented peptides are denoted as the immunogenic (dominant) and non-immunogenic (recessive) alleles, respectively. For the immune reaction to develop, the recipient should have at least one dominant allele, while the donor needs to have two copies of the recessive allele. For some MiHAs both alleles encode MHC-presented peptides, potentially making them immunogenic in both directions (co-dominant). In this case, T-cells discriminate peptides by a single amino acid difference (10). Up to date over $60 \mathrm{MiHAs}$ have been discovered. For the majority of their respective allelic counterparts, in vivo immunogenicity has not yet been confirmed; although for 36 alternative allelic variants of MiHAs, predicted HLA, HLA class I binding affinity is similar to the affinity of the respective MiHA (11). For a comprehensive review of currently known MiHAs and mechanisms of their immunogenicity see Griffioen et al. (12).

With a few exceptions, each MiHA is presented by only one HLA allele. Thus, for the immune response to occur, the donor and the recipient should not only differ in the allelic variants of MiHA-coding nsSNP but also have the restricting HLA allele. HLA-A*02:01 is the most frequent MHC class I allele in Caucasians, with up to $50 \%$ of individuals bearing this allele (13). Therefore, a substantial part of the patients undergoing HSCT in Europe and North America are HLA-A*02:01 positive. Approximately a third of the MiHAs discovered so far are restricted by the HLA-A*02:01. We selected 20 HLA-A*02:01restricted MiHAs for our study (Table $\mathbf{1}$ ).

Nonsynonymous SNPs can be genotyped by a vast arsenal of SNP-genotyping methods, with each having its limitations. The allele-specific PCR (AS-PCR) (28) and the analysis of restriction fragments length polymorphism (RFLP) (29) require an electrophoresis step to make the allele call. High resolution melting PCR (HRM-PCR) is hard to multiplex, could be unsuitable to certain SNP types and is prone to inaccuracies (30). qPCR with hydrolysis probes is accurate and fast, but the probe binding depends on the SNP allele. Most of the commercially available qPCR-based SNP genotyping kits are designed to genotype one SNP per test.

Sequence-based methods are expensive and time-consuming. The Sanger sequencing is the most accurate method, yet it lacks multiplexing. SNP genotyping could be scaled up by a single nucleotide extension reaction or by next-generation sequencing (NGS). Although NGS could be used to genotype many SNPs simultaneously, it is excessive for the small panel of known MiHAs. However, the NGS has been used for novel MiHA discovery $(22,31)$. SNP genotyping techniques are reviewed in
Kim and Misra (32). The genotyping of MiHAs is reviewed in Spierings and Goulmy (33).

Because the number of discovered MiHAs is limited, it is not practical to either genotype SNPs one by one or to use NGS. Besides, due to the HLA restriction, it is preferable to develop the genotyping panels grouped by the HLA allele. In this study, we aimed to design a straightforward, yet robust genotyping method based on a combination of the AS-PCR and the qPCR, for the largest group of MiHA-encoding polymorphisms, restricted by HLA-A*02:01. We designated it AS-qPCR. Our method is a significant improvement over the previously suggested panel for MiHA genotyping (28).

\section{MATERIALS AND METHODS DNA Samples}

Peripheral blood samples for genotyping were obtained from HSC recipients and their corresponding donors. Blood samples of healthy volunteers were used for cloning the control plasmids. All participants signed the informed consent approved by the ethical committee at the National Research Center for Hematology, Moscow, Russia. DNA was extracted using the Wizard Genomic DNA Purification Kit (Promega, USA).

\section{Primers and Probes Design}

Sequences spanning $500 \mathrm{bp}$ on either side of a target SNP were extracted from the Ensembl database (http://ensembl.org, Human genome assembly GRCh38) using in house made Perl script. Other polymorphisms with frequencies more than $1 \%$ according to the dbSNP database (http://ncbi.nlm.nih.gov/SNP/, build 150) were mapped to these sequences. The Geneious gene browser 4.8 (34) was used to manage sequences and for the primers and probes design. The ASP direction was picked according to the optimal GC content, Tm, low dimer and hairpin probability. The hydrolysis probes, when possible, were designed to anneal to the same strand as the ASP, between the ASP and the common primer. We tried to position the probe as close to the ASP $3^{\prime}$-end as possible, guanines at the $5^{\prime}$-end of the probe were avoided. Several ASP variants were designed for every MiHA with introduced deliberate mismatches 1 or 2 nucleotides from the $3^{\prime}$-end $(35,36)$. We tested all ASPs together with the gene-specific probes and the gene-specific primers using the control plasmids. For each MiHA we picked the ASPs with largest qPCR Cq difference between the target allele and the opposite allele. To avoid UGT2B17 paralog amplification both selected primers were sequence-specific, they flank the exon 6 fragment of the gene which encodes UGT2B17/A02 MiHA. All other SNPs, except MiHA-coding, were avoided during the primer and probe design. In the case of HA-8, it was impossible to avoid SNP at the probe binding site, so degenerate nucleotide was introduced. Also, for HA-1 and CCL4 obstructive SNPs (rs3764653 and rs1049807, respectively) were in close proximity to the target SNPs and they had to be included in the ASP. We checked the linkage disequilibrium for the SNPs of interest and the obstructing SNPs and found that in both cases adjacent SNPs were linked, so we designed the ASPs accordingly. All primers and probes were tested in silico 
TABLE 1 | HLA-A*02:01 restricted MiHAs, selected for genotyping panel.

\begin{tabular}{|c|c|c|c|c|c|c|c|c|}
\hline$\#$ & MiHA & Gene & Ch. & Nt. & AA & Pmm & Var. ID & References \\
\hline 1 & HER-2/NEU & ERBB2 & 17 & $\mathbf{C} / \mathrm{G}$ & $\mathbf{P} / \mathrm{A}$ & 0.247 & rs1058808 & $(14)$ \\
\hline 2 & $\mathrm{HA}-1 / \mathrm{A} 2$ & ARHGAP45 & 19 & G/A & $\mathrm{R} / \mathbf{H}$ & 0.246 & rs1801284 & $(15)$ \\
\hline 3 & $\mathrm{HA}-2$ & MYO1G & 7 & $\mathbf{c} / T$ & $\mathrm{~V} / \mathrm{M}$ & 0.050 & rs61739531 & (16) \\
\hline 4 & UTA2-1 & KIAA1551 & 12 & $\mathbf{T} / \mathrm{C}$ & $\mathbf{L} / P$ & 0.234 & rs2166807 & $(17)$ \\
\hline 5 & LB-ADIR-1F & TOR3A & 1 & $\mathbf{T} / \mathrm{C}$ & F/S & 0.250 & rs2296377 & (18) \\
\hline 6 & LB-CLYBL-1Y & CLYBL & 13 & $\mathrm{G} / \mathrm{T}$ & $\mathrm{D} / \mathbf{Y}$ & 0.056 & rs17577293 & (19) \\
\hline 7 & C19ORF48 & C19ORF48 & 19 & $\mathrm{~T} / \mathbf{A}$ & $T / \mathbf{S}$ & 0.082 & rs3745526 & (20) \\
\hline 8 & TRIM22 & TRIM22 & 11 & $\mathrm{C} / \mathbf{T}$ & $\mathrm{R} / \mathbf{C}$ & 0.019 & rs187416296 & (21) \\
\hline 9 & LB-PRCP-1D & $P R C P$ & 11 & $\mathrm{~T} / \mathbf{G}$ & $E / D$ & 0.226 & rs2229437 & (22) \\
\hline 10 & LB-SSR1-1S & SSR1 & 6 & $\mathrm{~A} / \mathbf{G}$ & $\llcorner/ \mathbf{S}$ & 0.246 & rs10004 & (22) \\
\hline 11 & LB-WNK1-1I & WNK1 & 12 & $\mathrm{G} / \mathrm{T}$ & $\mathrm{M} / \mathrm{I}$ & 0.237 & rs12828016 & (22) \\
\hline 12 & T4A1 & TRIM42 & 3 & C/A & A/E & 0.202 & rs9876490 & (23) \\
\hline 13 & $\mathrm{HA}-8$ & PUM3 & 9 & $\mathbf{C} / \mathrm{G}$ & $\mathbf{R} / \mathrm{P}$ & 0.206 & rs2173904 & (9) \\
\hline 14 & LB-HIVEP1-1S & HIVEP1 & 6 & $\mathrm{~A} / \mathbf{G}$ & N/S & 0.175 & rs2228220 & (24) \\
\hline 15 & LB-NISCH-1A & $\mathrm{NISCH}$ & 3 & $\mathbf{C} / \mathrm{T}$ & $\mathbf{A} / \mathrm{V}$ & 0.220 & rs887515 & (24) \\
\hline 16 & UGT2B17/A2 & UGT2B17 & 4 & & & 0.123 & esv3600873,4 & (25) \\
\hline 17 & LB-CCL4-1T & CCL4 & 17 & $\mathrm{~T} / \mathbf{A}$ & $\mathrm{S} / \mathbf{T}$ & 0.246 & rs1719152 & (26) \\
\hline 18 & LB-NCAPD3-1Q & NCAPD3 & 11 & $\mathrm{C} / \mathbf{T}$ & $\mathrm{R} / \mathbf{Q}$ & 0.130 & rs12292394 & (26) \\
\hline 19 & LB-NDC80-1P & NDC80 & 18 & G/C & $\mathrm{A} / \mathbf{P}$ & 0.241 & rs9051 & (26) \\
\hline 20 & WDR27-1L & WDR27 & 6 & $\mathbf{A} / \mathrm{G}$ & $\mathbf{L} / P$ & 0.205 & rs4236176 & $(27)$ \\
\hline
\end{tabular}

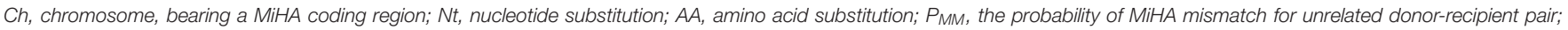

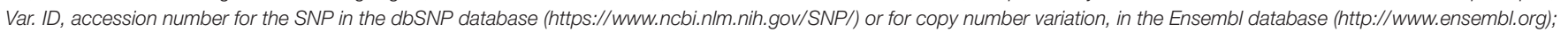
Ref., reference. The allelic variant and the corresponding amino acid, for which the immunogenicity is confirmed is shown in bold. UGT2B17/A2 is caused by gene deletion.

for dimer and hairpin formation using the IDT OligoAnalyzer tool with "qPCR" parameters setting (http://idtdna.com/calc/ analyzer). All primers were designed to have a $\mathrm{Tm}$ in the range of $63-68^{\circ} \mathrm{C}$ and the probes-in the range of $70-74^{\circ} \mathrm{C}$ according to the Multiple Primer Analyzer tool. Resulting primer pairs and probes were checked for mispriming on the human genome with the Primer-BLAST tool (http://ncbi.nlm.nih.gov/tools/primerblast/). Several primers were adapted from other studies studies, to some we introduced mismatches. The set of primers and probe, specific for beta-2 microglobulin (B2M), was used as the internal qPCR control. See Supplementary Table 1 for oligonucleotide sets and their source (if applicable). Overview of the primer positioning scheme is presented in Figure 1A.

\section{Control Plasmids}

The NCBI Primer-BLAST tool was used to design primers flanking the genotyping primers for cloning and sequence-based typing. In several cases, the common AS-qPCR primer was used for cloning. To clone the UGT2B17 exon six fragment we used the genotyping ASPs. First, we genotyped volunteers using AS-PCR without established positive control. For each cloning, preferably, homozygous DNA samples were selected. PCR for cloning was performed using the AccuPrime Pfx DNA polymerase (Thermo Fisher Scientific, USA) according to manufacturer's instructions. The list of cloning primers provided in Supplementary Table 2. The PCR products were purified using agarose gel electrophoresis and the GeneJET Gel Extraction Kit (Thermo Fisher Scientific, USA) and ligated into the pJet1.2 blunt vector with the CloneJET
PCR Cloning Kit (Thermo Fisher Scientific, USA). The plasmid DNA from the transformed $\mathrm{DH} 5 \alpha$ cells was extracted with the GeneJET Plasmid Miniprep kit (Thermo Fisher Scientific, USA). For the rare TRIM22 MiHA coding SNP allele we used mutagenic primers and cloning primers to perform two-step overlap extension PCR, its product was cloned as described (see Supplementary Table 3 for primer sets). All control plasmids were Sanger sequenced using standard pJet1.2 primers. The plasmids were mixed in the groups of 4 in a panel-wise manner (see below) with the concentrations equalized at $0.5-1 \mathrm{pg} / \mu \mathrm{l}$ to be used as the control for the genotyping panels.

\section{AS-QPCR}

The primers and probe set combinations were tested for heterodimer formation using the Multiple Primer Analyzer tool (Thermo Fisher Scientific, https://www.lifetechnologies. com) with the default settings. Genotyping sets were pooled into 5 panels of 4 least cross-reactive primer sets. The control set for $B 2 M$ was included in each pool. All panels were divided into 2 groups: with the ASPs for the reference and for the alternative SNP alleles, according to the reference human genome (http://ensembl.org, Human genome assembly GRCh38). The UGT2B17/A02 MiHA primers were the same for both oligonucleotide mixes. Table 2 shows the color channels assignment and the pooling scheme. For the CFX96 real-time PCR detection system (Bio-Rad, USA), used for the study, we have chosen FAM, HEX, ROX, Cy5, and Cy5,5 hydrolysis probe 


\section{A}

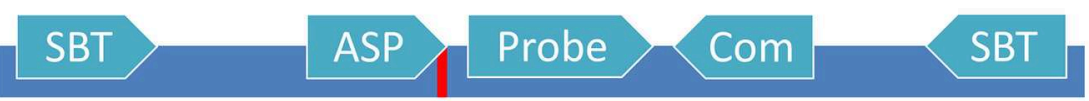

B

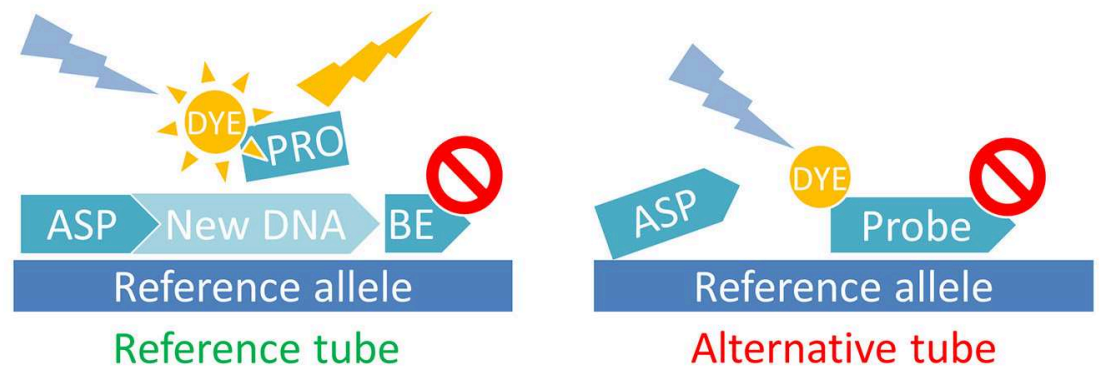

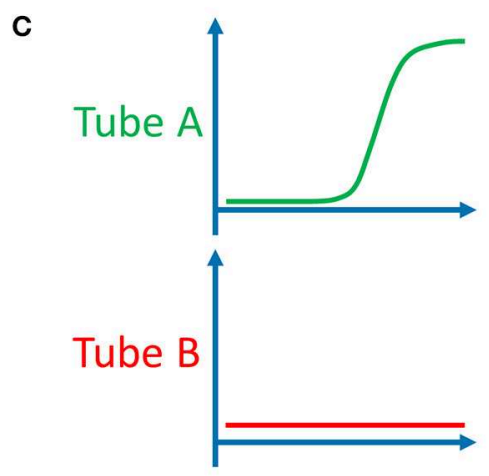

A/A

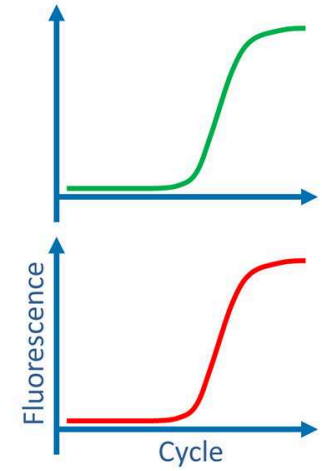

A/B

\section{Genotype calling}

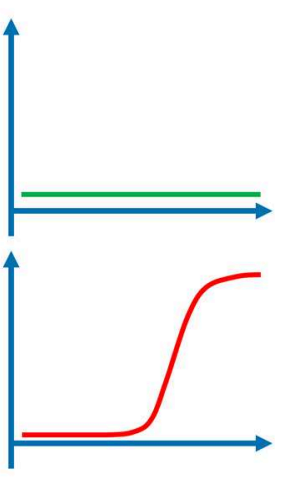

B/B

FIGURE 1 | Assay principle. (A) Schematic representation of the MiHA-coding locus with all the utilized oligonucleotides. SBT, sequence-based typing primers used for Sanger sequencing and for control plasmids cloning; ASP, allele-specific primers used for the AS-qPCR genotyping, complementary with the $3^{\prime}$-end nucleotide to each of the SNP alleles (indicated by red line); Probe, a hydrolysis probe, bearing fluorescent dye and quencher; Com, common primer, used for both SNP alleles AS-qPCRs (for some SNPs common primer was used as the second SBT primer). For UGT2B17/A2 the ASP primers were used as the SBT primers. (B) Schematic representation of the AS-qPCR reaction. The assay is performed in 2 separate tubes with different ASPs for the same SNP. Each tube contains the common gene-specific primer (not shown) and the gene-specific fluorescent probe. Here is represented the genotyping of a DNA sample homozygous for a reference allele. (C) Schematic representation of the possible outcomes of the AS-qPCR and their interpretation. Allele calls are listed below the graphs.

dyes for color channels $1-5$, respectively. The BHQ-1 quencher was used for the FAM and HEX labeled probes and the BHQ-2 for the remaining dyes. Primers and probes were synthesized by EvroGen or Syntol (Russia).

$300 \mathrm{nM}$ of each primer and $200 \mathrm{nM}$ of each probe (150 and $100 \mathrm{nM}$ for the control set, respectively) were used for the reaction. Ready-made 5x qPCRmix-HS PCR mix (EvroGen, Russia) containing Taq polymerase was used to make the stock reaction solutions. The qPCRmix-HS qPCR mix provides $3 \mathrm{mM}$ $\mathrm{Mg}^{2+}$ and $0.2 \mathrm{mM}$ of each $\mathrm{dNTP}$ in the final $10 \mu \mathrm{l}$ reaction. The AS-qPCRs were performed using two-step qPCR protocol: for $2 \mathrm{~min}$, then 40 cycles of alternated $98^{\circ} \mathrm{C}$ for $10 \mathrm{~s}$ and $61^{\circ} \mathrm{C}$ for $30 \mathrm{~s}$ with plate read. The method scheme is shown in Figure 1B.

\section{Analysis}

The SNP calling was performed using the CFX Manager 3.1 software (Bio-Rad, USA). Quantification cycle (Cq) was used to evaluate the AS-qPCR results, the $\mathrm{Cq}<30$ was considered as positive (37). We also evaluated the $B 2 M$ fluorescence curve shape to check for possible evaporation. Related wells for the same sample and for the same MiHAs were evaluated simultaneously, target by target, starting with the internal control evaluation-respective B2M Cqs should be $<1.5$ cycles apart. The SNP allele calling was performed according to the scheme in Figure 1C. If both PCRs were positive-the sample was marked as heterozygous, if only one well had positive signal or the signal plot crossed the threshold two or more cycles ahead of the other well-the sample was designated homozygous. Samples with the fluorescence level below 300 relative fluorescence units (RFU) were not taken into account. Both wells for the UGT2B17 locus contain the same oligo set for that gene, so only the bi-allelic deletion will render both reactions negative.

For plots in Figure 2 raw qPCR data were extracted in the CSV format, fluorescent curves were plotted using GraphPad Prism version 5.03 (GraphPad Software, USA, www.graphpad.com), it was also used for Supplementary Figures 1-3.

The probability of MiHA Mismatch $\left(\mathrm{P}_{\mathrm{MM}}\right)$ for related and unrelated donor-recipient pairs was calculated using the 


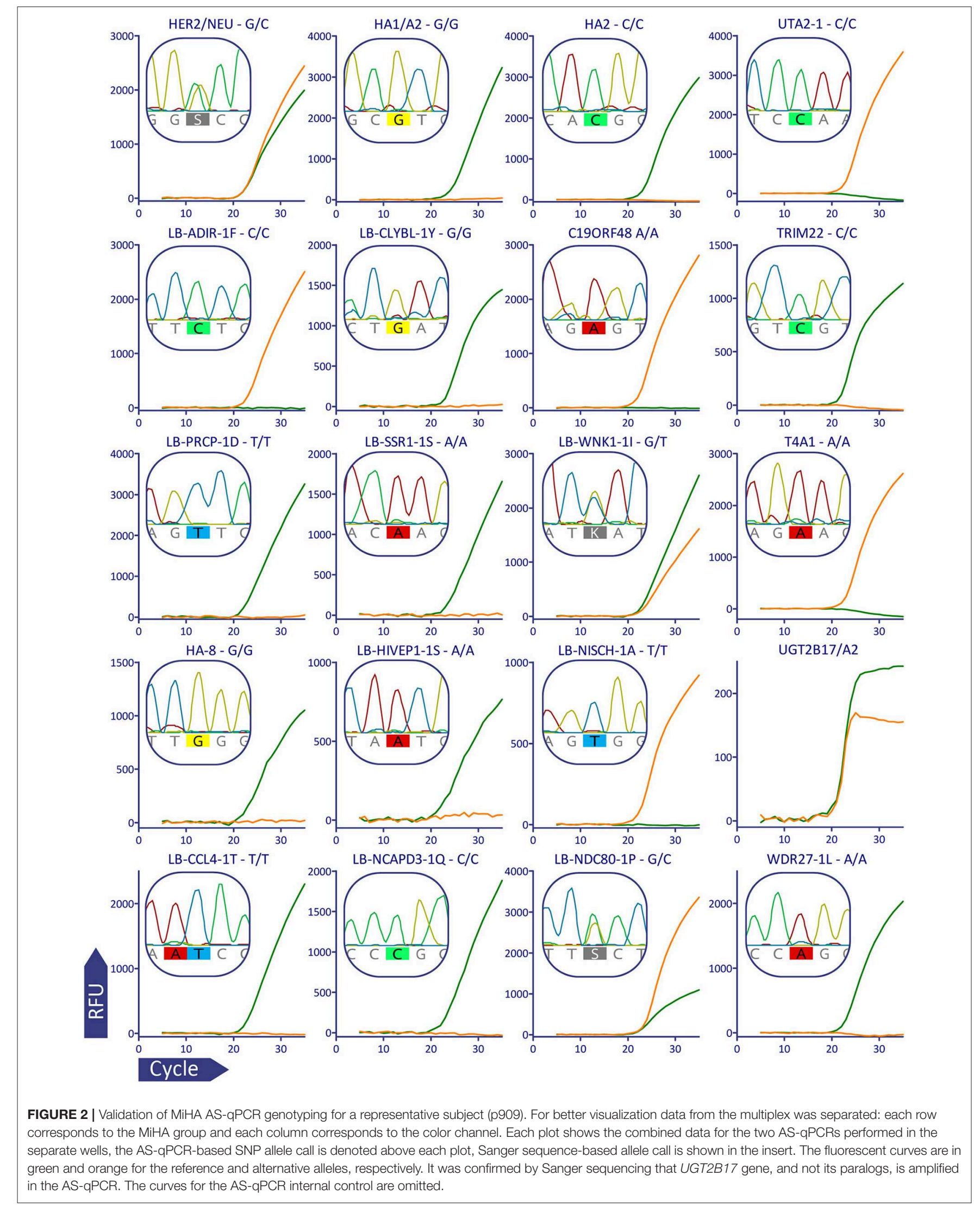


TABLE 2 | MiHA genotyping mixes. Primer sets are grouped into 5 multiplex panels.

\begin{tabular}{|c|c|c|c|c|c|}
\hline \multirow[t]{2}{*}{ Color channel } & \multicolumn{5}{|c|}{ Panels } \\
\hline & I & II & III & IV & $\mathbf{v}$ \\
\hline 1 & HER-2/NEU & LB-ADIR-1F & LB-PRCP-1D & HA-8 & LB-CCL4-1T \\
\hline ॥ & $\mathrm{HA}-1 / \mathrm{A} 2$ & LB-CLYBL-1Y & LB-SSR1-1S & LB-HIVEP1-1S & LB-NCAPD3-1Q \\
\hline III & $\mathrm{HA}-2$ & C19ORF48 & LB-WNK1-1I & LB-NISCH-1A & LB-NDC80-1P \\
\hline IV & UTA2-1 & TRIM22 & $\mathrm{T} 4 \mathrm{~A} 1$ & UGT2B17/A2 & WDR27-1L \\
\hline V & $B 2 M$ & $B 2 M$ & $B 2 M$ & $B 2 M$ & $B 2 M$ \\
\hline
\end{tabular}

Color channel, QPCR machine filter sets. FAM, HEX, ROX, Cy5, and Cy5.5 dyes were used for first to fifth channels.

SNP allele frequencies for European population from the reference genome database (http://ensembl.org, Human Genome Assembly GRCh38) according to the formulas provided in Bykova et al. (38), Armistead et al. (23). The distribution of the number of the mismatches was calculated by $10^{7}$ iterations of simulation with an in-house python script.

\section{Sequencing}

The PCRs were performed with the 5x qPCRmix-HS PCR mix (EvroGen, Russia) or the Phusion Hot Start II DNA Polymerase (Thermo Fisher Scientific, USA) using the cloning primers. The product was checked with agarose gel electrophoresis and PCR was enzymatically cleaned using the Illustra ExoStar kit (GE Healthcare, USA) or with electrophoresis and the gel-extraction Cleanup kit (EvroGen, Russia). The sequencing reactions were performed with the BigDye 1.1 or 3.1 chemistry, purified with the BigDye XTerminator (Applied Biosystems, USA) and sequenced using the Nanophor-05 (Syntol, Russia) or the ABI PRISM 3100 (Applied Biosystems, USA) machines. Base calling was performed using the Sequencing analysis software version 5.3 (Applied Biosystems, USA). Obtained reads were aligned with the respective reference sequences, described above, using the Geneious software version 4.8. Chromatogram images were made using Geneious version R11 (34).

The exome libraries were made using the Ion AmpliSeq Exome RDY Kit $1 \times 8$ (Thermo Fisher Scientific, USA) for 8 donor-recipient pairs. Exome sequencing was performed at the Research Center for Medical Genetics, Moscow, Russia using the Ion S5 system (Thermo Fisher Scientific, USA). Exome assembly was performed using the Torrent Suite software (Thermo Fisher Scientific, USA). The MiHA coding polymorphisms were analyzed using the Integrative Genomics Viewer (39). The data points with coverage below 15 reads were excluded, MiHAs C19ORF48 and LB-NDC80-1P were fully excluded due to this criterion. For the UGT2B17 exon six coverage was analyzed.

\section{RESULTS}

\section{HLA-A*02:01-Restricted MiHA Panel}

For our study, we have selected 20 previously described HLA-A*02:01 restricted MiHAs (Table 1). Most of them were discovered by the forward immunology approaches, so their ability to induce the in vivo immune response was confirmed. To our best knowledge, for HER2/Neu, HIVEP1-1S, NISCH-1A,
UGT2B17/A2, and WDR27-1L only the in vitro immunogenicity was shown. UGT2B17/A2 MiHA is caused by gene deletion, so it lacks an allelic counterpart, for the other MiHAs, encoded by SNPs, both peptides are translated. For HA-1, HA-2 and HA8 it was shown that the alternative peptides are not presented by the MHC due to impaired HLA-A*02:01 binding or altered antigen processing $(9,15,29)$. For LB-CLYBL-1Y, LB-SSR1-1S, NDC80-1P, and LB-NISCH-1A it was demonstrated by massspectrometry that the allelic counterpart was presented by HLA$\mathrm{A}^{*}$ 02:01 molecules $(10,27)$. If mismatched, they may elicit an immune response, but in the current study we called mismatch only in the cases where the recipient had the allele known to be immunogenic in vivo and the donor was homozygous for the alternative allele. Although UGT2B17/A2 was initially reported to be presented by HLA-A*02:06 (40), it is assumed that it may also be presented by HLA-A*02:01, as these alleles have similar peptide binding motifs (41). The peptide was labeled as a weak binder for both HLA-A*02:01 and $A^{*} 02: 06$ alleles by the NetMHC 4.0 algorithm (42).

The probability of a donor and recipient to have a MiHA mismatch $\left(\mathrm{P}_{\mathrm{MM}}\right)$ for a particular MiHA depends on the allele frequencies $(23,38)$. $\mathrm{P}_{\mathrm{MM}}$ in the European population ranges from $2 \%$ for TRIM22 to $25 \%$ for LB-ADIR-1F (Table 1). Using $\mathrm{P}_{\mathrm{MM}}$ for each MiHA, we have calculated the distribution of the number of mismatches in related and unrelated pairs for the $20 \mathrm{MiHAs}$ considered in this paper (Supplementary Figure 1). Related allo-HSCT-pairs were most likely to have 2 mismatches $(28.8 \%)$, while the number of mismatches in unrelated pairs peaks at $3(22.9 \%)$. This confirms the clinical relevance of the genotyping for the selected panel, as most transplantations would be mismatched for one or more of the studied MiHAs.

\section{AS-qPCR Assay Design}

To achieve PCR multiplexing and to reduce the analysis time we used the combination of allele-specific PCR and real-time PCR: the allele-specific primers were used to discriminate SNP alleles while the fluorescently labeled hydrolysis probes distinguished the loci (Figure 1). With the proposed design, the genotyping of four SNPs required two separate wells: one for the detection of the reference and other for the alternative alleles. Each had the fifth, common oligonucleotide set, serving as the internal control. This design allowed for the 4-fold reduction of the number of reactions compared to a singleplex AS-PCR and the 
2-fold reduction compared to a singleplex qPCR with allelespecific probes.

The $\Delta$ Cq between the specific and the non-specific reaction for ASPs of our design was at least 4.9 (Supplementary Figure 2). Using the plasmid mixes, we checked the sensitivity of the allele discrimination to the amount of input DNA across the series of dilutions from $100 \mathrm{pg}$ to $10 \mathrm{fg}$ of plasmid DNA (pDNA) per test, which approximated to $80 \mu \mathrm{g}$ and $0.8 \mathrm{ng}$ of human genomic DNA (gDNA), respectively. We found that although in the multiplex reaction with the 5x qPCRmix-HS qPCR mix some MiHAs can be genotyped using as low as $10 \mathrm{fg}$ of $\mathrm{pDNA}$, reliable results were obtained with at least $1 \mathrm{pg}$ of pDNA (Supplementary Figure 3). This corresponds to $80 \mathrm{ng}$ of human gDNA.

\section{Method Validation}

To validate the genotyping panel, we selected 5 HLA-matched HSCT donor-recipient pairs bearing the HLA-A*02:01 allele (1 sibling donor and 4 unrelated donors). All samples were genotyped in a blind manner with the reported method and using Sanger sequencing. In all of the 200 tested data points, Sanger sequencing confirmed the allele calls made with the AS-qPCR. Figure 2 shows qPCR curves and Sanger sequencing data for a representative subject, the rest of the genotyping data could be found in the Supplementary Dataset.

To further test our approach, we performed the full exome sequencing for 8 additional HLA-matched HSCT donorrecipient pairs on the Ion S5 platform. We found that some SNPs in our panel were poorly covered by the full exome sequencing (Supplementary Figure 4). Altogether, 42 points had to be excluded from the analysis due to low coverage, including all data points for C19ORF48 and LB-NDC80-1P. The remaining 278 points were compared to AS-qPCR genotyping data. We found the discrepancies in 5 cases. The SNP encoding the LBWNK1-1I was wrongly genotyped in subject p1032 due to the rare SNP rs56245971 located 7 nucleotides upstream from the target SNP, which interfered with the ASP binding, leading to the wrong allele call. The interfering SNP was not taken into account during the design process, due to its low frequency of $0.005 \%$ (according to The Exome Aggregation Consortium, http://exac. broadinstitute.org). The remaining 4 genotyping errors were contained in panel 4 (3 for HA-8, and 1 for LB-HIVEP1-1S). We assume that they were caused by the mistake in the preparation of the genotyping mixes resulting in low signal levels. We repeated the AS-qPCR for this panel in the same blind manner and found no discrepancies. Taking this into consideration we propose that the test results with the signal level below 300 RFU should not be taken into account.

Sibling pairs have $0-5$ mismatches and unrelated pairs -0 to 8 mismatches. The donor-recipient genotypes and the imputed mismatches are listed in Table 3.

\section{DISCUSSION}

Here we report the method for genotyping $20 \mathrm{MiHA}$-encoding polymorphisms based on AS-PCR combined with qPCR. This approach is faster than the conventional AS-PCR, lacks the electrophoresis step and could be multiplexed. We demonstrated that up to 4 AS-qPCRs, plus the internal control, can be performed in a single tube without the loss of accuracy, and the test is robust on the wide range of gDNA concentrations.

Current work describes the genotyping of all currently known HLA-A*02:01-restricted MiHAs. There could be other MiHAs presented by HLA-A*02:01 yet to be discovered. Besides, other alleles, including HLA- $A^{*} 01: 01, A^{*} 03: 01, A^{*} 24: 02$, $B^{*} 07: 02, B^{*} 08: 01$, and $B^{*} 44: 02$, which are common in the European population, have associated MiHAs. We believe that this approach could be further extended to all immunogenic polymorphisms. Designing the genotyping kits based on the MiHA-restricting HLA allele, in our opinion, is more practical than whole exome sequencing or large SNP-genotyping panels.

The limitation of the reported approach is shared with other methods that use ASPs, i.e., previously unknown or rare polymorphisms falling into primer binding sites may affect the results. Indeed, using Sanger sequencing, we discovered a novel SNP in the ASP binding site for SNP rs9876490 (T4A1) in the sibling pair p908/p909. However, as both donor and patient were homozygous for the genotyped SNP, the novel polymorphism did not preclude the correct allele call. In subject p1032 analyzed by NGS, we found a rare SNP in the ASP binding site for LB-WNK11I. This SNP led to the wrong allele call by our method. These risks should be taken into consideration, but due to the high number of rare variants, it seems impractical to consider them during ASP design.

We aimed to identify mismatches that could induce the immune response in the genotyped donor-recipient pairs. For HA-1, HA-2, and HA-8 $(9,15,29)$ it was demonstrated that only one of the allelic variants yielded an MHC-associated peptide, other MiHAs in this work may be immunogenic in both directions (co-dominant). In a recent study using quantitative mass-spectrometry, it was demonstrated that the allelic counterparts of LB-CLYBL-1Y, LB-NISCH-1A, and LB-SSR1-1S were presented by the MHC at comparable levels to the MiHA-encoding alleles (10). The in vivo immunogenicity of the alternative allelic variants still needs to be confirmed. In our assay the allelic variants are grouped according to the reference human genome and not by immunogenicity. In this way, the proposed method would remain applicable irrespective of the notion of MiHA-immunogenicity.

MiHA can contribute to the outcome of HSCT. Autosomal MiHA mismatches increased the incidence of relapse-free survival after HLA-matched sibling transplantations (43). It was recently reported that mismatches for two HLA$\mathrm{A}^{*}$ 02:01-restricted MiHAs: HA-1 and HA-8 increased the incidence of severe acute GvHD when the donor had A/A genotype in rs231775 of CTLA4 gene (44). The contribution of mismatches of the other MiHAs to the clinical outcome has not yet been demonstrated. The availability of the assay allowing for rapid MiHA genotyping of donor-recipient pairs should facilitate the study of the allogeneic immune response directed against MiHAs. Besides, the proposed approach can be easily adapted for genotyping other DNA polymorphisms, including SNPs in immunoregulatory genes. 
TABLE 3 | MiHA genotyping for 13 HLA-matched HSCT pairs,

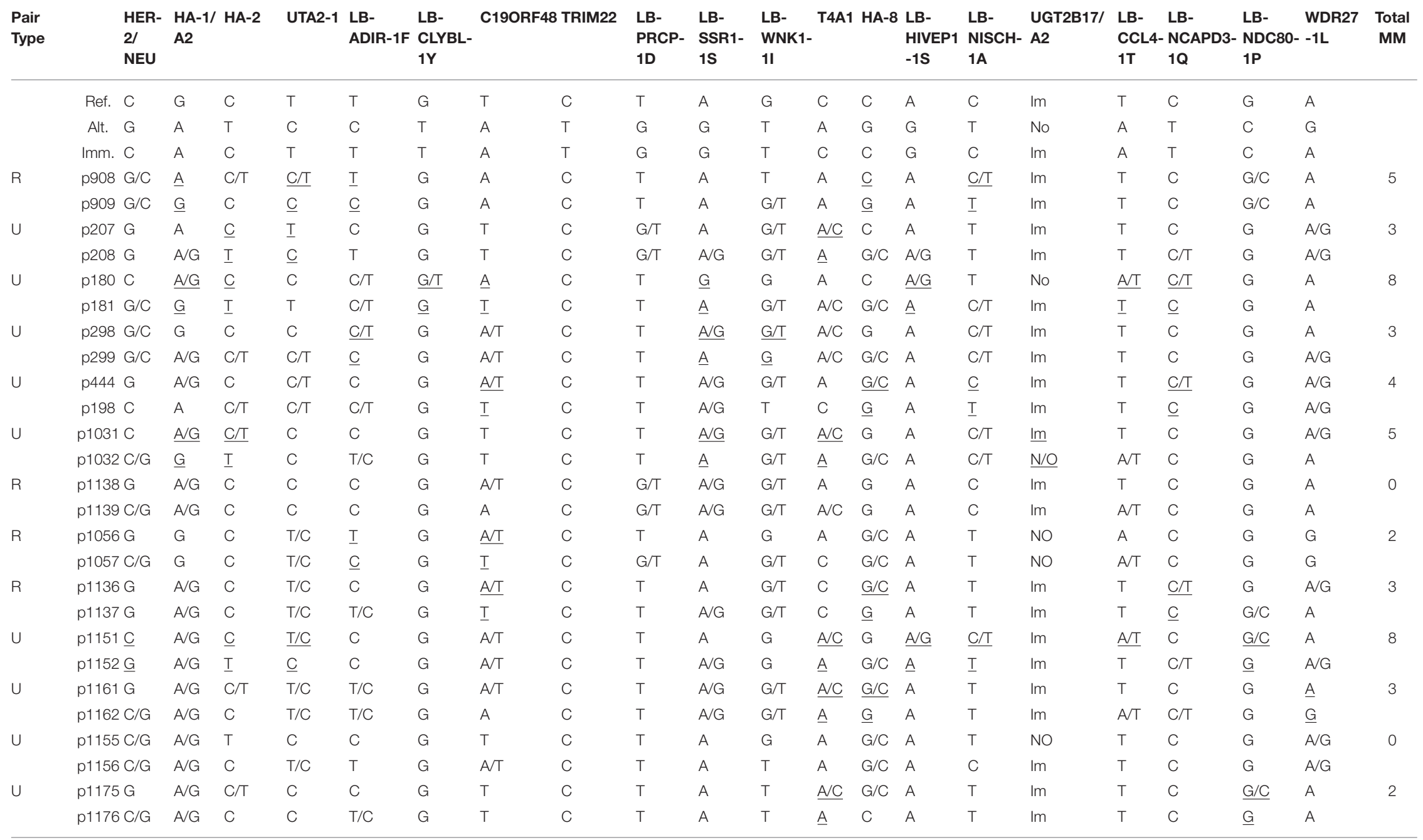

Ref, reference; Alt, alternative nsSNP allele; according to the human reference genome (http://ensembl.org, Human Genome Assembly GRCh38), Imm, the allelic variant with confirmed immunogenicity. Recipients and corresponding donors are listed in pairs, with patients above the donors. R, sibling; $U$, unrelated donor. The immunogenic mismatches underlined. Total MM, the number of immunogenic mismatches for a given HSCT pair. 
Another possible application of MiHA genotyping is a selection of therapeutic targets for post-transplant immunotherapy. Up to $58 \%$ of patients relapse post-HSCT (45). MiHAs represent the attractive targets for posttransplant cell therapy as, unlike tumor neoantigens, they are germlineencoded and relatively common in the population, so all cancer cells, expressing MiHA-encoding gene, can be targeted. To avoid the potential off-tumor toxicity, immune therapy should be restricted only to MiHAs encoded by the genes selectively or predominantly expressed in the hematopoietic tissue (5). The AS-qPCR could be used for the preliminary patient and donor screening for the targetable MiHA mismatches.

We hope that the reported method will foster research of the allogeneic immune response and development of the novel immunotherapies.

\section{ETHICS STATEMENT}

The study was conducted in accord with the Declaration of Helsinki (1964). All subjects participated in the study signed a written informed consent approved by the ethical committee at the National Research Center for Hematology, Moscow, Russia.

\section{REFERENCES}

1. Falkenburg FJ, Warren EH. Graft versus leukemia reactivity after allogeneic stem cell transplantation. Biol Blood Marrow Transpl. (2011) 17:S33-8. doi: 10.1016/j.bbmt.2010.11.009

2. Shlomchik WD. Graft-versus-host disease. Nat Rev Immunol. (2007) 7:nri2000. doi: 10.1038/nri2000

3. Neefjes J, Jongsma ML, Paul P, Bakke O. Towards a systems understanding of MHC class I and MHC class II antigen presentation. Nat Rev Immunol. (2011) 11:823. doi: 10.1038/nri3084

4. de Bueger M, Bakker A, Van Rood JJ, Van der Woude F, Goulmy E. Tissue distribution of human minor histocompatibility antigens. Ubiquitous versus restricted tissue distribution indicates heterogeneity among human cytotoxic T lymphocyte-defined non-MHC antigens. J Immunol. (1992) 149:1788-94.

5. Bleakley M, Riddell SR. Exploiting $\mathrm{T}$ cells specific for human minor histocompatibility antigens for therapy of leukemia. Immunol Cell Biol. (2011) 89:396-407. doi: 10.1038/icb.2010.124

6. Feng X, Hui K, Younes HM, Brickner AG. Targeting minor histocompatibility antigens in graft versus tumor or graft versus leukemia responses. Trends Immunol. (2008) 29:624-32. doi: 10.1016/j.it.2008.09.004

7. de Rijke B, van Horssen-Zoetbrood A, Beekman JM, Otterud B, Maas F, Woestenenk R, et al. A frameshift polymorphism in P2X5 elicits an allogeneic cytotoxic $\mathrm{T}$ lymphocyte response associated with remission of chronic myeloid leukemia. J Clin Invest. (2005) 115:3506-16. doi: 10.1172/JCI24832

8. Spierings E, Brickner AG, Caldwell JA, Zegveld S, Tatsis N, Blokland E, et al. The minor histocompatibility antigen HA-3 arises from differential proteasome-mediated cleavage of the lymphoid blast crisis (Lbc) oncoprotein. Blood. (2003) 102:621-9. doi: 10.1182/blood-2003-01-0260

9. Brickner A, Warren E, Caldwell J, Akatsuka Y, Golovina T, Zarling A, et al. The immunogenicity of a new human minor histocompatibility antigen results from differential antigen processing. J Exp Med. (2001) 193:195-206. doi: $10.1084 / \mathrm{jem} .193 .2 .195$

10. Bijen HM, Hassan C, Kester MG, Janssen GM, Hombrink P, Ru AH, et al. Specific $\mathrm{T}$ cell responses against minor histocompatibility antigens cannot generally be explained by absence of their allelic counterparts on the cell surface. Proteomics. (2018) 18:1700250. doi: 10.1002/pmic.201700250

11. van der Lee DI, Pont MJ, Falkenburg FJ, Griffioen M. The value of online algorithms to predict $\mathrm{t}$-cell ligands created by genetic

\section{AUTHOR CONTRIBUTIONS}

DR designed the method. DR, AP, and AK performed method validation and genotyping. DM performed statistical calculations and data analysis. DR and GE wrote the manuscript. All authors read and reviewed the manuscript.

\section{ACKNOWLEDGMENTS}

The work was supported by the Russian Science Foundation grant 17-15-01512. The bioinformatics was supported by the Russian Foundation for Basic Research project No.17-04-02186. Authors would like to thank Vladimir V. Strelnikov and Alexander S. Tanas for help with performing the NGS, and Nadia A. Bykova, and Dasha Efimova for the critical reading of the manuscript.

\section{SUPPLEMENTARY MATERIAL}

The Supplementary Material for this article can be found online at: https://www.frontiersin.org/articles/10.3389/fimmu. 2019.01226/full\#supplementary-material variants. PLoS ONE. (2016) 11:e0162808. doi: 10.1371/journal.pone. 0162808

12. Griffioen $M$, van Bergen CA, Falkenburg J. Autosomal minor histocompatibility antigens: how genetic variants create diversity in immune targets. Front Immunol. (2016) 7:100. doi: 10.3389/fimmu.2016. 00100

13. González-Galarza FF, Takeshita L, Santos E, Kempson F, Maia M, Silva A, et al. Allele frequency net 2015 update: new features for HLA epitopes, KIR and disease and HLA adverse drug reaction associations. Nucleic Acids Res. (2015) 43:D784-8. doi: 10.1093/nar/gku1166

14. Wenandy L, Kollgaard T, Letsch A, Andersen R, Stather D, Seremet T, et al. The $1170 \mathrm{~A}-\mathrm{P}$ single-nucleotide polymorphism (SNP) in the Her-2/neu protein (HER2) as a minor histocompatibility antigen (mHag). Leukemia. (2009) 23:leu2009112. doi: 10.1038/leu.2009.112

15. den Haan J. The minor histocompatibility antigen HA-1: a diallelic gene with a single amino acid polymorphism. Science. (1998) 279:1054-7. doi: 10.1126/science.279.5353.1054

16. Haan DJ, Sherman NE, Blokland E, Huczko E, Koning F, Drijfhout $J$, et al. Identification of a graft versus host disease-associated human minor histocompatibility antigen. Science. (1995) 268:1476-80. doi: $10.1126 /$ science. 7539551

17. Oostvogels R, Minnema M, van Elk M, Raapen, te Raa G, Giovannone B, et al. Towards effective and safe immunotherapy after allogeneic stem cell transplantation: identification of hematopoietic-specific minor histocompatibility antigen UTA2-1. Leukemia. (2013) 27:642. doi: 10.1038/leu.2012.277

18. van Bergen CA, Kester MG, Jedema I, Heemskerk MH, van Luxemburg-Heijs $\mathrm{SA}$, Kloosterboer FM, et al. Multiple myeloma-reactive T cells recognize an activation-induced minor histocompatibility antigen encoded by the ATPdependent interferon-responsive (ADIR) gene. Blood. (2007) 109:4089-96. doi: 10.1182/blood-2006-08-043935

19. Hombrink P, Hassan C, Kester MG, Jahn L, Pont MJ, de Ru AH, et al. Identification of biological relevant minor histocompatibility antigens within the B-lymphocyte-derived HLA-ligandome using a reverse immunology approach. Clin Cancer Res. (2015) 21:2177-86. doi: 10.1158/1078-0432.CCR-14-2188

20. Tykodi SS, Fujii N, Vigneron N, Lu SM, Mito JK, Miranda MX, et al. C19orf48 encodes a minor histocompatibility antigen recognized by $\mathrm{CD} 8+$ cytotoxic $\mathrm{T}$ 
cells from renal cell carcinoma patients. Clin Cancer Res. (2008) 14:5260-9. doi: 10.1158/1078-0432.CCR-08-0028

21. Wölfel C, Lennerz V, Lindemann E, Hess G, Derigs HG, Huber C, et al. Dissection and molecular analysis of alloreactive CD8+ T cell responses in allogeneic haematopoietic stem cell transplantation. Cancer Immunol. Immunother. (2008) 57:849-57. doi: 10.1007/s00262-0070421-1

22. Bergen CA, Rutten CE, Meijden ED, Luxemburg-Heijs SA, Lurvink EG, Houwing-Duistermaat JJ, et al. High-throughput characterization of 10 new minor histocompatibility antigens by whole genome association scanning. Cancer Res. (2010) 70:9073-83. doi: 10.1158/0008-5472.CAN-10-1832

23. Armistead PM, Liang S, Li H, Lu S, Bergen CA, Alatrash G, et al. Common minor histocompatibility antigen discovery based upon patient clinical outcomes and genomic data. PLoS ONE. (2011) 6:e23217. doi: 10.1371/journal.pone.0023217

24. Hombrink P, Hassan C, Kester MG, de Ru AH, van Bergen CA, Nijveen $\mathrm{H}$, et al. Discovery of $\mathrm{T}$ cell epitopes implementing HLA-peptidomics into a reverse immunology approach. J Immunol. (2013) 190:3869-77. doi: 10.4049/jimmunol.1202351

25. Murata M, Warren EH, Riddell SR. A human minor histocompatibility antigen resulting from differential expression due to a gene deletion. $J$ Exp Med. (2003) 197:1279-89. doi: 10.1084/jem.20030044

26. van Bergen CA, van Luxemburg-Heijs SA, de Wreede LC, Eefting M, von dem Borne PA, van Balen P, et al. Selective graft-versus-leukemia depends on magnitude and diversity of the alloreactive T cell response. JClin Invest. (2017) 127:517-29. doi: 10.1172/JCI86175

27. Granados D, Rodenbrock A, Laverdure J-P, Côté C, Caron-Lizotte O, Carli $\mathrm{C}$, et al. Proteogenomic-based discovery of minor histocompatibility antigens with suitable features for immunotherapy of hematologic cancers. Leukemia. (2016) 30:1344. doi: 10.1038/leu.2016.22

28. Spierings E, Drabbels J, Hendriks M, Pool J, Spruyt-Gerritse M, Claas F, et al. A uniform genomic minor histocompatibility antigen typing methodology and database designed to facilitate clinical applications. PloS ONE. (2006) 1:e42. doi: 10.1371/journal.pone. 0000042

29. Pierce RA, Field ED, Mutis T, Golovina TN, Kap-Herr C, Wilke. M, et al. The HA-2 minor histocompatibility antigen is derived from a diallelic gene encoding a novel human class I myosin protein. J Immunol. (2001) 167:3223-30. doi: 10.4049/jimmunol.167.6.3223

30. Słomka M, Sobalska-Kwapis M, Wachulec M, Bartosz G, Strapagiel D. High resolution melting (HRM) for high-throughput genotyping-limitations and caveats in practical case studies. Int J Mol Sci. (2017) 18:2316. doi: $10.3390 /$ ijms 18112316

31. Kawase T, Nannya Y, Torikai H, Yamamoto G, Onizuka M, Morishima $\mathrm{S}$, et al. Identification of human minor histocompatibility antigens based on genetic association with highly parallel genotyping of pooled DNA. Blood. (2008) 111:3286-94. doi: 10.1182/blood-2007-10-1 18950

32. Kim S, Misra A. SNP genotyping: technologies and biomedical applications. Ann Rev Biomed Eng. (2007) 9:289-320. doi: 10.1146/annurev.bioeng.9.060906.152037

33. Spierings E, Goulmy E. Molecular typing methods for minor histocompatibility antigens. Methods Mol Med. (2007) 134:81-96. doi: 10.1007/978-1-59745-223-6_7

34. Kearse M, Moir R, Wilson A, Stones-Havas S, Cheung M, Sturrock S, et al. Geneious Basic: an integrated and extendable desktop software platform for the organization and analysis of sequence data. Bioinformatics. (2012) 28:1647-9. doi: 10.1093/bioinformatics/bts199
35. Stadhouders R, Pas SD, Anber J, Voermans J, Mes T, Schutten M. The effect of primer-template mismatches on the detection and quantification of nucleic acids using the $5^{\prime}$ nuclease assay. J Mol Diagn. (2010) 12:109-17. doi: 10.2353/jmoldx.2010.090035

36. Little S. Chapter 9:9.8.1-9.8.12. In Haines JL, Korf BR, Moir DT, Morton CC, Seidman CE, Seidman JG, et al. editors. Current Protocols in Human Genetics/Editorial Board (2001)

37. Bustin SA, Benes V, Garson JA, Hellemans J, Huggett J, Kubista M, et al. The MIQE guidelines: minimum information for publication of quantitative real-time PCR experiments. Clin Chem. (2009) 55:611-22. doi: 10.1373/clinchem.2008.112797

38. Bykova NA, Malko DB, Efimov GA. In silico analysis of the minor histocompatibility antigen landscape based on the 1000 Genomes Project. Front Immunol. (2018) 9:1819. doi: 10.3389/fimmu.2018.01819

39. Robinson JT, Thorvaldsdóttir H, Wenger AM, Zehir A, Mesirov JP. Variant review with the Integrative Genomics Viewer. Cancer Res. (2017) 77:e31-4. doi: 10.1158/0008-5472.CAN-17-0337

40. Kamei M, Nannya Y, Torikai H, Kawase T, Taura K, Inamoto Y, et al. HapMap scanning of novel human minor histocompatibility antigens. Blood. (2009) 113:5041-8. doi: 10.1182/blood-2008-07-171678

41. Sudo T, Kamikawaji N, Kimura A, Date Y, Savoie C, Nakashima H, et al. Differences in MHC class I self peptide repertoires among HLA-A2 subtypes. J Immunol. (1995) 155:4749-56.

42. Andreatta M, Nielsen M. Gapped sequence alignment using artificial neural networks: application to the MHC class I system. Bioinformatics. (2016) 32:511-7. doi: 10.1093/bioinformatics/btv639

43. Dzierzak-Mietla M, Markiewicz M, Siekiera U, Mizia S, Koclega A, Zielinska $\mathrm{P}$, et al. Occurrence and impact of minor histocompatibility antigens' disparities on outcomes of hematopoietic stem cell transplantation from HLA-matched sibling donors. Bone Marrow Res. (2012) 2012:257086. doi: 10.1155/2012/257086

44. Gallardo D, Bosch-Vizcaya A, Rodríguez-Romanos R, Santos N, Buño I, de la Cámara $\mathrm{R}$, et al. donor CTLA-4 genotype modulates the immune response to minor histocompatibility antigen mismatches. Biol Blood Marrow Transpl J Am Soc Blood Marrow Transpl. (2017) 23:2042-7. doi: $10.1016 /$ j.bbmt.2017.08.003

45. D'Souza A, Fretham C. The Summary Slides are an annual report on data submitted to the CIBMTR by centers worldwide and describes information related to practices and general survival outcomes after hematopoietic cell transplantation. The Current Edition Includes Transplants Performed Prior to 2017. (2017) Available online at: http://www.cibmtr.org

Conflict of Interest Statement: Patent application No.2017122175 describing the current method was filed with the Russian Patent Office. DR, AP, and GE had filed a Russian patent application, RU 2675597 C1 describing the current method.

The remaining authors declare that the research was conducted in the absence of any commercial or financial relationships that could be construed as a potential conflict of interest.

Copyright (ङ 2019 Romaniuk, Postovskaya, Khmelevskaya, Malko and Efimov. This is an open-access article distributed under the terms of the Creative Commons Attribution License (CC BY). The use, distribution or reproduction in other forums is permitted, provided the original author(s) and the copyright owner(s) are credited and that the original publication in this journal is cited, in accordance with accepted academic practice. No use, distribution or reproduction is permitted which does not comply with these terms. 